http://dx.doi.org/10.18778/2196-8403.2013.06

ANNA DASZKIEWICZ

\title{
Struwwelpeter, Anti-Struwwelpeter und Złota różdżka. Ein pädagogisches Bilderbuch zum „Leviten lesen“ im Vergleich
}

Niniejszy artykuł zwraca uwagę na fenomen książki obrazkowej, której dobrym przykładem jest pionierska praca HeInRICHA Hoffmanna Der Struwwelpeter. Słowo i obraz - jako równoważne elementy całego tekstu - mają tu za zadanie skłonić dzieci do posłuszeństwa i grzecznego zachowania. Wśród niektórych autorów książek obrazkowych zachodzi jednakże rozbieżność w zakresie tego, jakie zachowanie należy uznać za właściwe, co znajduje wyraz w ich przekazie słownym i obrazkowym. Przykłady tego odnajdujemy w Der Anti-Struwwelpeter i Złotej Różdżce, zestawionymi tu w analizie porównawczej z prototypem w postaci tekstu Hoffmanna. Ponadto, niniejszy artykuł przedstawia zbiór dodatkowych komentarzy studentów germanistyki Uniwersytetu Gdańskiego jako odbiorców wspomnianych tu pozycji. Przytoczone wypowiedzi dotyczą związków pomiędzy tekstem a obrazem w prototypie i kolejnych jego wersjach.

Im vorliegenden Artikel wird das narrative Verfahren des Bilderbuches erörtert. Hier kommt HeInRICH Hoffmanns Struwwelpeter die Rolle eines Vorreiters zu. Wort und Bild sollen als gleichrangige Bestandteile des Textganzen Kinder zu Gehorsam und bravem Verhalten animieren. Gleichzeitig verweisen sie auf die Einstellung des Verfassers zur Regulierung von kindlichem Verhalten. Allerdings herrscht unter Bilderbuchautoren Uneinigkeit darüber, was als musterhaftes Verhalten einzustufen ist. Dies schlägt sich entsprechend in der Wort- und Bilddarstellung nieder. Beispiele hierfür bieten Der Anti-Struwwelpeter und Złota różdżka, die mit dem ursprünglichen Struwwelpeter-Text verglichen werden. Zusätzlich enthält der Artikel Kommentare von Studierenden der Universität Gdańsk zu den Text-Bild-Bezügen in den besprochenen Struwwelpeter-Fassungen.

This article addresses the phenomenon of the picture book, a good example of which is a pioneering work by HeInRICH Hoffmann, Der Struwwelpeter. The word and the 
picture - as equally important components of the entire text - are meant to cause children to remain obedient. Among some authors, however, there appears discrepancy as to what conduct should be viewed as proper, which is reflected in their textual and graphic message. The examples of this we can find in, for instance, Der AntiStruwwelpeter and Złota Różdzka, which have been juxtaposed here in a comparative analysis with Hoffmann's work. Moreover, the text presents a collection of additional comments made by students of German Studies at the University of Gdańsk as recipients of the said works. The remarks cited concern relationships between the text and picture in the prototype and its subsequent versions.

Am 5. September 1983 bat der Kulturminister der DDR eine Anzahl maßgeblicher Personen um die Benennung von zehn Buchtiteln, deren Vorliegen für die DDR von Nutzen sein könnte. Ich antwortete am 11. September 1983. Und gleich mein Punkt 1 lautete: ,Heinrich Hoffmann: Struwwelpeter (Das beste Bilderbuch der Welt)‘. Die Wichtigkeit [des Struwwelpeters] ist für mich in erster Linie eine ästhetische Wichtigkeit, keine psychologische. Der Text ist gleichermaßen glänzend wie die Bilder, und das graphische Miteinander von Text und Bild ist überhaupt nicht wieder erreicht. (HACKS in: HOFFMANN 2010:34)

Aufgrund der Annahme, dass Kinder wie Erwachsene durch Beobachtung, Imitation und Teilhabe lernen, greifen Struwwelpeter-Vater Heinrich Hoffmann und seine Nachfolger auf die Technik des Bilderbuchs ${ }^{1}$ zurück. Sie huldigen damit der These, dass die Neugierde und Phantasie der Kinder dann am besten angeregt und ihre Selbstständigkeit an nützlichen Dingen geübt wird, wenn sie über ihre bevorzugte Lektüre literarischen Anschauungsunterricht erhalten. Dieser Anschauungsunterricht kann als Hilfe zur Selbsthilfe ausgelegt werden, d.h., er ermöglicht es, sich selbst als Mittelpunkt der heterogenen Welt zu erfahren und ein Subjekt in ihr zu werden. Er geht mit dem seit der Aufklärung (genauer gesagt seit Rousseaus Emile) bevorzugten Er-

1 Vgl. SCHWEIKLE / SCHWEIKLE (1990:53): „Bilderbuch, illustriertes Kinderbuch (für ca. 2- bis 8-jährige) mit farbigen, oft künstler. gestalteten Bildern. Entsprechend den jeweiligen Altersstufen bieten die Bilderbücher einfache Gegenstände (und Tiere) aus der Erfahrungs- und Phantasiewelt des Kindes (ohne Text), Bildergeschichten oder Illustrationen zu längeren Texten (zum Vorlesen). Die Texte (Kinderreime, Lieder, Verse und Prosa) entsprechen dem jeweiligen Erziehungsprogramm und reichen von moralisierender Belehrung über phantasievolle Märchen bis zu sachl. Information. Die Geschichte des B.s fällt bis ins 19. Jh. zus. mit der der illustrierten Kinder- u. Jugendlit.“ 
ziehungsstil einher, Kinder und junge Leute nicht mehr mit unverstandenem Wissen vollzustopfen und mit Memorieren zu langweilen, sondern ihnen durch bebilderte moralische Erzählungen eine Möglichkeit zur Besinnung auf ihre eigenen Denk- und Verhaltensweisen zu bieten (vgl. HERRMANN 1993:14f.). Die doppelte Kraft des Bilderbuchs, das Wörtliche durch das Illustrierte und umgekehrt auszudrücken sowie auf diese Weise zu unterstützen, soll demnach Kinder fesseln und sie zu Einsicht und Nachdenken in Bezug auf das eigene Handeln anhalten. Als Beispiel für die Auswirkung des Kinderbuchs habe ich mich in meinem Artikel an HeINRICH HoffMAnNs Der Struwwelpeter (2010), FRIEDRICH KARL WAECHTERs Der Anti-Struwwelpeter (1982) sowie an der polnischen Fassung des Struwwelpeters, Złota różdżka $(1933)^{2}$, orientiert. Diesen Ausgaben sind sowohl die im Beitrag zitierten Textpassagen als auch die Illustrationen entnommen. Im Anschluss an meine Ausführungen veranschauliche ich diese anhand einer Untersuchung zur Text-Bild-Rezeption von Studierenden der Germanistik an der Universität Danzig.

Mit dem vorliegenden Artikel möchte ich zum einen auf das durch das Bilderbuch ermöglichte und realisierte Erziehungsprogramm hinweisen, zum anderen jedoch die im Bilderbuch stets vorhandene Überschneidung von Wort und Bild thematisieren. Mir ist bewusst, dass das Thema im Rahmen dieses Beitrags lediglich skizziert, keinesfalls aber erschöpfend behandelt werden kann. Es wäre jedoch von Interesse, die im Beitrag diskutierte Problematik in einer größeren Arbeit zu vertiefen.

\section{Zu HEINRICH HofFMANNs Der Struwwelpeter}

Hoffmanns Hauptimpuls, ein Bilderbuch zu schreiben, war, seinem dreijährigen Sohn Carl ein Weihnachtsgeschenk zu machen. Dass aus seinem Geschenk eines der berühmtesten Bilderbücher der Weltliteratur wurde ${ }^{3}$, konnte

2 Zur besseren Unterscheidung verweise ich auf die polnische Übersetzung unter dem Namen des Übersetzers, WACŁAW NowAKOWSKI, nicht des Autors - A. D.

3 Vgl. dazu CARCENAC-LECOMTE (2009:130): „Der Struwwelpeter hat sehr viele Nachahmer auf den Plan gerufen, und nur Robinson Crusoe von Daniel Defoe kann mit dieser Zahl an Variationen - den Robinsonaden - mithalten. Allerdings hat wohl kein anderes Kinderbuch so viele politische Adaptionen erfahren wie der Struwwelpeter.“ Vgl. auch HERzog / HERzOG-HoinKIS / SiEfERT (1995:71): „Es gibt kein zweites Kinderbuch auf der Welt, das eine so große Verbreitung gefunden hat. [...] Der ,Struwwelpeter` (näml. der ursprüngliche und echte) ist 
er nicht ahnen. Als sich der Autor um Weihnachten 1844 auf die Suche nach einem Bilderbuch machte, fand er keines, das „der Fassungskraft des kleinen menschlichen Wesens in solchem Alter entsprechend schien“ (STOLLE 1871:769). Es standen damals nur „lange Geschichten“, „alberne Bildersammlungen“ oder „moralische Geschichten“ zur Verfügung, die „mit ermahnenden Vorschriften“ wie beispielsweise „Das brave Kind muss wahrhaft sein“ oder „Brave Kinder müssen sich reinlich halten“ begannen und schlossen (STOLLE 1871:769). Hoffmann war vom damaligen Angebot an Kinderbüchern enttäuscht. Die Geschichten mit ihren ermahnenden Vorschriften erschienen ihm zu lang, die Bildersammlungen dagegen zu albern. Doch Hoffmanns Frustration in Bezug auf das unzureichende Angebot verweist zugleich darauf, was er selbst als kindgerechten Text versteht und welche Funktionen dieser Text zu erfüllen hat: Er sollte weder lang noch langweilig sein, dabei jedoch zum Denken und zur Reflexion anregen und die selbstständige Analyse fördern. Hoffmann lehnt es jedoch ab, fertige Rezepte zu geben, er bevorzugt es, wenn Kinder einem Problem selbst auf den Grund gehen. Der Struwwelpeter-Autor argumentiert: „Abstrakt denkt ja das Kind noch gar nicht, und die allgemeine Warnung: ,Du sollst nicht lügen!‘ hat wenig ausgerichtet im Vergleich mit der Geschichte: ,Fritz, Fritz, die Brücke kommt!““ (STOLLE 1871:769) Damit wird offenbar, dass für Hoffmann ein Text die kindliche Vorstellungskraft auf möglichst effektive Weise ansprechen sollte und dass Wort und Bild im gleichen Maße für die Botschaft eines Textganzen arbeiten. Denn zu Recht konstatiert HoffMANN: „Mit der absoluten Wahrheit, mit algebraischen oder geometrischen Sätzen rührt man [aber] keine Kinderseele, sondern läßt sie elend verkümmern. [...] Dem Kinde ist ja alles wunderbar, was es schaut und hört, und im Verhältnis zum immer noch Unerklärten ist überhaupt die Masse des Erkannten doch auch nicht so gewaltig.“ (Zit. nach STOLLE 1871:770 - Hervorh. A. D.)

kein ,dummes Zeug', sondern ,der Struwwelpeter‘ gehört zu den Klassikern der Kleinen. Es wird in der ganzen Welt kein Kind geben, das nicht am ,Struwwelpeter' eine unendliche Freude hätte. Ergo ist der ,Struwwelpeter' ein gutes Kinder-Buch, denn der Geschmack der Kinder ist unverdorben. Hüten wir uns, den Geschmack der unverdorbenen Kinder durch unsern Geschmack, der nicht mehr so unschuldig und kindlich ist, korrigieren zu wollen. Sie sind kompetente Richter auf ihrem Gebiet, nicht wir Große.“ Zu den bekanntesten Adaptionen zählen u. a. Die Struwwelliese von LÜTJE / MADDALENA (1890), Struwwelhitler (SPENCE / SPENCE 1941), Anti-Struwwelpeter (WAECHTER 1970) oder auch Der tierische Struwwelpeter (GROSCHE 2007). 
Obwohl der Text- und Bilddarstellung im Allgemeinen dieselbe appellative und erzieherische Funktion zukommt, können Rezipienten ihre Anziehungskraft unterschiedlich wahrnehmen. Nicht auszuschließen ist auch, dass das Bild dem Text seine ursprüngliche Kraft nehmen kann, denn präzise angefertigte Illustrationen scheinen, selbst wenn sie keinen Text enthalten, die kindliche Aufmerksamkeit ausreichend zu bündeln und Phantasie zu wecken. Dies führt schließlich dazu, dass das tragende Element der StruwwelpeterStruktur vor allem das Bild, weniger der Text ist. ${ }^{4}$ Die tragende Kraft der Zeichnungen sieht auch Hoffmann, wenn er sagt, Kinder könnten sich besser merken, was sie „sehen“ und „hören“ (zit. nach STOLLE 1871:770). Nicht weniger ist er jedoch davon überzeugt, dass gerade das zwischen den Zeilen Geschriebene, das nicht in Worte Gefasste und somit sich nicht Aufdrängende die Tiefen des kindlichen Bewusstseins am stärksten anspricht und geeignet ist, das Kind zur selbstständigen Entscheidung in Bezug auf sein Verhalten zu befähigen. ${ }^{5}$

Das Hauptthema, das im Struwwelpeter-Text nach und nach entfaltet wird, ist der Trotz bzw. das kindliche Unartigsein. Schon im Vorwort macht HofFMANN (2010:7) seine Leser darauf aufmerksam:

Wenn die Kinder artig sind,

Kommt zu ihnen das Christkind; [...]

Bringt es ihnen Gut's genug

Und ein schönes Bilderbuch.

Was er unter Artigsein versteht, erläutert Hoffmann noch im Vorwort (das Personalpronomen „sie“ referiert auf die oben angesprochenen Kinder):

Wenn sie ihre Suppe essen

Und das Brot auch nicht vergessen,

Wenn sie, ohne Lärm zu machen,

Still sind bei den Siebensachen,

$4 \quad$ Vgl. dazu die im Artikel angegebenen Untersuchungsergebnisse.

5 Vgl. dazu STOLLE (1871:770): „Als ich nun gar endlich ein Foliobuch fand, in welchem eine Bank, ein Stuhl, ein Topf und vieles andere, was wächst oder gemacht wird, ein wahres Weltrepertorium, abgezeichnet war, und wo bei jedem Bild fein säuberlich zu lesen war: die Hälfte, ein Drittel oder ein Zehntel der natürlichen Größe - da war es mit meiner Geduld aus. Einem Kinde, dem man eine Bank zeichnet und sich daran erfreuen soll, ist dies eine Bank, eine wirkliche Bank. Und von der wirklichen Lebensgröße der Bank hat und braucht das Kind gar keinen Begriff zu haben.“ 
Anna Daszkiewicz

Beim Spaziergehn auf den Gassen

Von Mama sich führen lassen.

Weil Kinder jedoch das Artigsein nicht selten verweigern, wie Hoffmanns Helden Kamm und Schere verabscheuen (der Struwwelpeter), die Nahrungsaufnahme verweigern (der Suppen-Kaspar), gegen den elterlichen Willen mit Streichhölzern spielen (Paulinchen) oder leichtsinnig (Hans Guck-in-dieLuft) und ungehorsam (der Zappel-Philipp, der fliegende Robert) sind, müssen sie mit Strafe rechnen. Durch ihr Schicksal sollen die Helden des Struwwelpeters Kinder einerseits vor den Folgen ihres Verhaltens warnen, andererseits aber bei der Charakterformung Hilfestellung leisten. Die Darstellung der kindlichen Hauptfiguren samt ihren wunderlichen Geschichten soll bewirken, dass Rezipierende für sich selbst ein besseres Los anstreben und sich von den im Struwwelpeter personalisierten Verhaltenstypen möglichst distanzieren sowie schließlich einen Gegenentwurf erarbeiten:

In psychologischer Sicht könnte hier von ,splitting‘ die Rede sein, da sich das Kind ,teilt‘ und das negative Ich in die Figur des Kindes projiziert, das in den Geschichten für seinen Ungehorsam bestraft wird, so dass es die Strafe nicht selbst erleiden muss. Denn die Daumen werden ja dem Daumenlutscher abgeschnitten und der Suppenkaspar wird am Ende sterben, nicht das Kind, das diese Schauergeschichten liest und am Ende froh das Buch schließt, da es heil bleiben kann. (BURWICK 2010:142)

Man sollte allerdings nicht außer Acht lassen, dass HoFFMANNs (2010:13, 23) Helden das schlimmste Unheil widerfährt, wenn (oder vielleicht, weil) ihre Eltern abwesend sind:

Paulinchen war allein zu Haus,

Die Eltern waren beide aus.

Fort geht nun die Mutter, und

Wupp! Den Daumen in den Mund. //

Bauz! Da geht die Türe auf,

Und herein in schnellem Lauf

Springt der Schneider in die Stub،

Zu dem Daumen-Lutscher-Bub.

Weh! Jetzt geht es klipp und klapp

Mit der Scher' die Daumen ab [...].

Die Kluft, die die Welt der Kinder und die der Eltern voneinander trennt, ist spürbar. Diese Distanz wird im Text zum einen durch den Gebrauch der Äußerung „die Frau Mama“ (Die Geschichte vom Daumenlutscher), zum anderen durch den des bestimmten Artikels wie bei „der Philipp“ (Die Geschichte vom Zappel-Philipp) vollzogen: 


\author{
,Ob der P h i l i p heute still \\ Wohl bei Tische sitzen will? \\ Also sprach in ernstem Ton \\ Der Papa zu seinem Sohn [...]. \\ (HofFMAnN 2010:25 - Hervorh. A. D.)
}

Die endgültige Unmöglichkeit, in die Welt der Erwachsenen einzudringen, kommt wohl am deutlichsten durch Konrads Mutter zum Ausdruck:

„Konrad!‘ sprach die Frau Mama,

,ich geh aus und du bleibst da.[']

(HOFFMANN 2010:22)

Mit diesen Worten wird zugleich auf die grundverschiedenen Rollen beider Generationen verwiesen: Der Vertreter der Kinderwelt, Konrad, hat auf seine Mutter zu hören, ihr absolut zu gehorchen, er darf ermahnt und trotz seiner Machtlosigkeit, die sich im Daumenlutschen ausdrückt, weiter eingeschüchtert werden. Auch Philipp, der wegen seiner Hyperaktivität auch ZappelPhilipp genannt wird, werden elterliche Zuneigung und Verständnis vorenthalten. Statt Unterstützung und Aufmerksamkeit erfährt er vom Vater Zorn und von der Mutter Gleichgültigkeit. Zwar wird in beiden Geschichten nicht explizit benannt, dass die Eltern ihre Kinder ermahnen, ihnen drohen oder sie einschüchtern, darauf verweisen jedoch die suggestiven Zeichnungen. Sie spiegeln die Emotionen der Figuren wider und ersetzen hier eine verbale Abbildung:

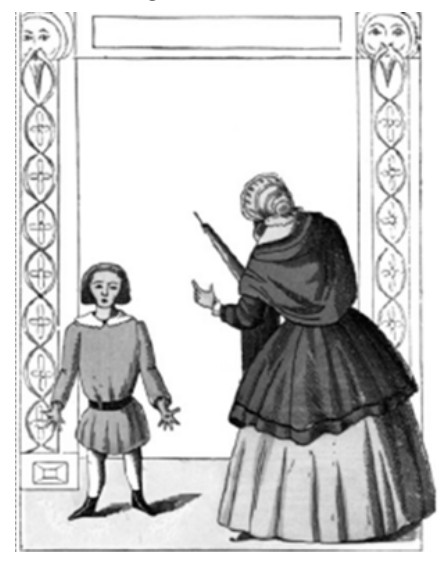

(HoFFMANN 2010:22)

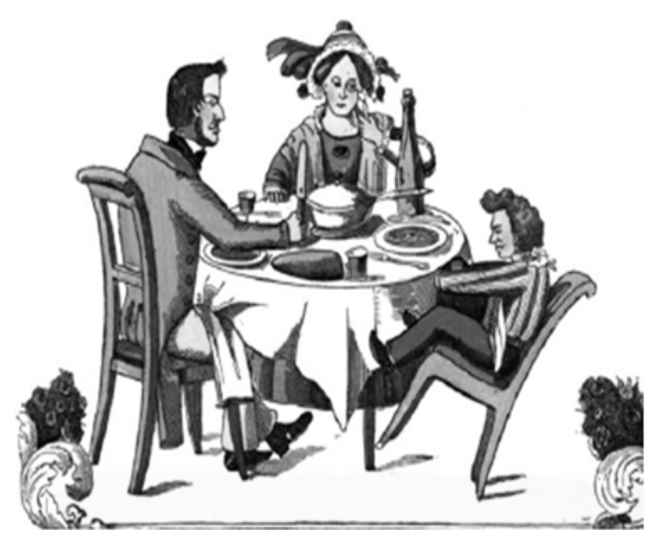

(HOFFMANN 2010:25) 
Daraus ist ersichtlich, dass die Mutter des daumenlutschenden Kindes den autoritären Erziehungsstil verkörpert:

Wer sähe sie nicht vor sich, so die zum Ausgang gekleidete Madame, drohend mit dem Zeigefinger und mit der erhobenen Schirmspitze, die verkörperte erzieherische Gewalt. Und das kläglich ihr gegenüberstehende Kind, schon im Voraus schuldbewusst, weil es die Trennung von der Mutter, die Verlassenheit nicht anders wird überstehen können als mit dem tröstenden Daumen im Mund. Und schon, kaum ist die Mutter fort, kommt der Schneider herein - wer hat ihn nur geschickt? Wie ein Tanz, ein grauenvolles Ballett ist die Szene dargestellt, in der die trostspendenden Daumen mit einer Riesenschere grausam abgeschnitten werden. Und dann steht es vor uns, das furchtbar bestrafte, für seine Schwäche noch verstümmelte Kind, ein Bild des Jammers. (FLITNER 2009:9 - Hervorh. A. D.)

Welchen Erfolg die Erwachsenenfiguren im Struwwelpeter mit ihrem autoritären Erziehungsstil tatsächlich haben, ist fraglich; statt den Trotz ihrer Kinder im Keim zu ersticken, verstärken sie den Zustand der Unruhe und Ratlosigkeit bei ihren Kindern nur noch mehr. Sie tragen dadurch zur Verstümmelung oder zum Tod ihrer Kinder bei. Bemerkenswert ist, dass die meisten Kinderfiguren im Struwwelpeter in den Elementen, also Feuer, Wasser, Erde und Luft, ums Leben kommen. REIMAR KLEIN (2005:79) analysiert die Bilddarstellung der Texte und bemerkt, dass auch der Zappel-Philipp unter dem Tischtuch verschwinde und somit der elterlichen Dominanz ausweiche: „Auf der Erde rollt aber auch Philipp, der Urheber des Desasters. Er ist präsent, aber nicht mehr identifizierbar; nachdem er den Eltern das Essen entzogen hat, entzieht er sich ihren Blicken: Das Tischtuch, das ihn nicht halten konnte, kann ihn wenigstens verbergen.“ Das Verschwinden der Kinder aus der Erwachsenenwelt steht zugleich für ihre Befreiung. Deutlich wird dies an der Figur des fliegenden Roberts. Robert darf seinen Flug genießen und andere Handlungsräume erleben: „Aber Wolken und Regen sind im Grunde nur Staffagen, die Robert bald hinter sich läßt; sein wahres Abenteuer verschafft ihm der Sturm. ,Pfeifend und keuchend‘ bietet er alles auf, um aus dem einzelgängerischen Kind ein weltflüchtiges zu machen.“ (KLEIN 2005:93) Der fliegende Robert kann sich in seinem Glück völlig ungefährdet fühlen, darf seinen Flug ungestört fortsetzen (also seine Existenz ungehindert führen) und ist unerreichbar:

Schirm und Robert fliegen dort durch die Wolken immer fort.

Und der Hut fliegt weit voran, stößt zuletzt am Himmel an.

Wo der Wind sie hingetragen, 
Struwwelpeter, Anti-Struwwelpeter und Zlota różdżka

ja! das weiß kein Mensch zu sagen.

(HOFFMANN 2010:31)

Der Schaden der geraubten Kindheit ist nicht einschätzbar. Die Kinder erleben nicht, welchen Schaden es anrichtet, dass man ihnen das Kindsein nimmt. Sie erfahren nicht, was Freiheit bedeutet. Vielmehr übernehmen sie eher unbewusst das Verhalten der Eltern, lernen absolute Unterwürfigkeit ihnen gegenüber und verzichten allmählich auf eine eigene Identität. Dabei haben nicht nur Erwachsene, sondern auch Kinder das Recht auf ein eigenes Weltbild. Dieses Recht darf niemandem genommen werden. Denn dann ist das Heranwachsen zur selbstständigen, selbstbewussten und sozial ausgeglichenen Persönlichkeit nicht möglich.

\section{Złota różḋ̇ka ${ }^{6}$ - Zur polnischen Fassung des Struwwelpeters}

Die polnische Bearbeitung des Struwwelpeters setzt die im deutschen Original dargelegte Denkweise über Kindererziehung fort. Zwar wird in der polnischen Fassung die Abfolge von Hoffmanns Geschichten nicht beibehalten, doch auch in ihr konzentriert sich die Bildaussage auf die Darstellung von Strafe. Damit wird die Haltung, Kinder benötigten den pädagogischen Zeigefinger und man müsse ihnen durch disziplinierende Maßnahmen Gehorsam abverlangen, betont. Während im Original die Bestrafung überwiegend nicht durch die Eltern, sondern eine unkontrollierbare Macht erfolgt, maßregeln die Eltern in der polnischen Version durchaus selbst. Dabei ist die Palette der Strafen breit gefächert und reicht von Ermahnungen über Drohungen bis hin zur Züchtigung. Ein Beispiel dafür ist die Geschichte Pawełek Wiercipięta. Während in der Geschichte vom Zappel-Philipp der Titelheld lediglich unter der Folge seines trotzigen Verhaltens leidet und seine Niederlage in der Isolation (er ist unter der Tischdecke versteckt) besteht, wird seinem polnischen Pendant Pawełek Wiercipięta nicht nur ein Glas auf dem Kopf zerschlagen,

$6 \quad$ Die zum ersten Mal im polnischen Text vorkommende Diminutivform różdżka bedeutet ,Wünschelrute‘. Hätte man aber in diesem Fall auf die Diminutivform verzichtet, hätte der Titel pejorative Züge, denn die nicht diminuierte Form, róz$g a$, bedeutet ,Rute' und wird in erster Linie mit Bestrafung assoziiert. Die irreführende Wirkung des Titels besteht außerdem darin, dass die Geschichten keineswegs in einer magischen Welt, sondern vielmehr in der Wirklichkeit angesiedelt sind. Das wiederum ist ein Hinweis darauf, dass man sich bei der Auslegung des Titels auf eine andere Deutungs- und Argumentationsart stützen muss. 
sondern er wird auch vom Vater unter der Tischdecke hervorgezogen und in Anwesenheit seiner Mutter heftig verprügelt. Dass beide Figuren für denselben Unfug ungleich behandelt und bestraft werden, bleibt als Unterscheidungsmerkmal hervorstechend.
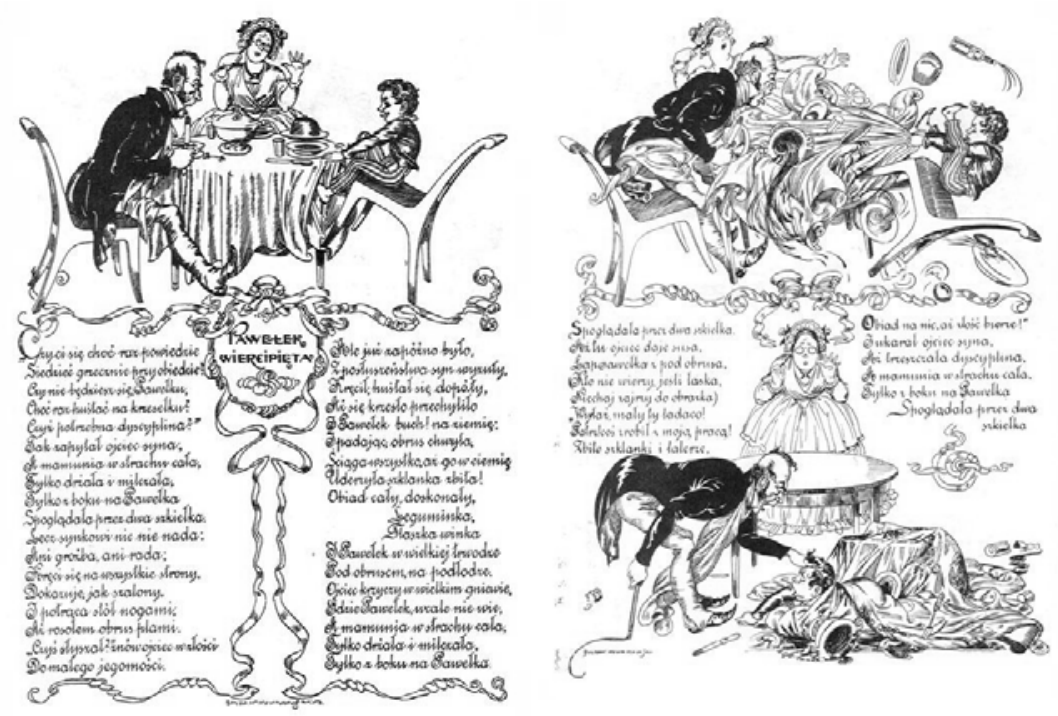

(NoWAKOWSKI 1993: Pawetek Wiercipięta)

In beiden Geschichten reagiert die Mutter jeweils anders auf die Geschehnisse am Tisch. Während die Mutter des Zappel-Philipps regungslos dasitzt und nur auf den Tisch starrt, scheint die Mutter von Pawełek Wiercipięta über das Verhalten ihres Sohnes erregt zu sein. Das ist zumindest an ihrer Körpersprache abzulesen. Sie nimmt zwar nicht am Gespräch zwischen Vater und Sohn teil, ihre Hände befinden sich aber nicht mehr unter, sondern über dem Tisch, was im Widerspruch zur Haltung der Mutter des Zappel-Philipps steht. Auch die Mutter von Juleczek (der in der polnischen Textversion für den Daumen lutschenden Konrad steht) lässt ihren Sohn ihre mütterliche Zuwendung erfahren. Erstens erfährt der Junge, warum seine Mutter geht (sie geht seinetwegen in die Stadt, um ihm Kekse zu kaufen). Zweitens darf er sich zwar beim Abschied und während ihrer Abwesenheit nicht mit Daumenlutschen trösten, aber er darf zumindest wissen, warum das so ist: Das Daumenlutschen sei unanständig und müsse rigoros bestraft werden. Drittens wird Jule- 
czeks Mutter auch bildlich anders gezeigt als die Mutter des Daumenlutschers. Während die Protagonistin Hoffmanns im Halbprofil und mit weggedrehtem Kopf dargestellt wird (und so durch den Schutenhut nichts von ihrem Gesicht zu sehen ist), wendet sich die Mutter von Juleczek nicht nur ihrem Sohn zu, sondern auch der Leserschaft (was möglicherweise die Reinheit ihrer Absichten bestätigt). Deckungsgleich ist in beiden Fällen die Ermahnung mit dem erhobenen Zeigefinger, doch statt wie Konrads Mutter die zweite Hand auf einem Schirm abzustützen, streichelt Juleczeks Mutter ihrem Sohn sanft über den Kopf. Mit dieser vielsagenden Geste bekräftigt sie die starke Mutter-Kind-Beziehung:

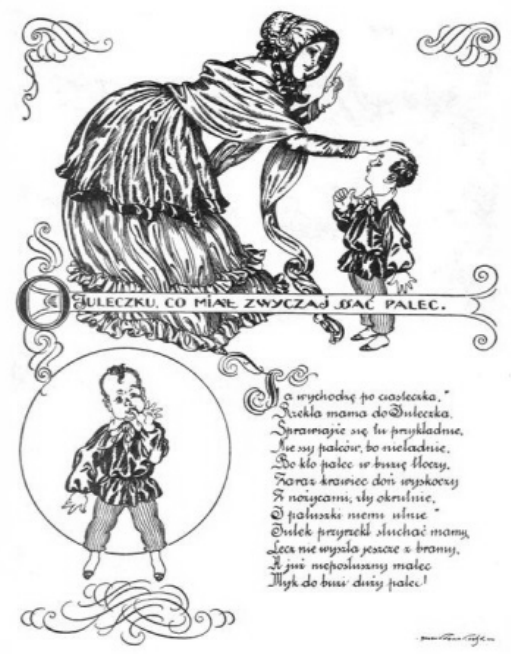

(NowAKOWSKI 1933: O Juleczku, co miat zwyczaj ssać palec)

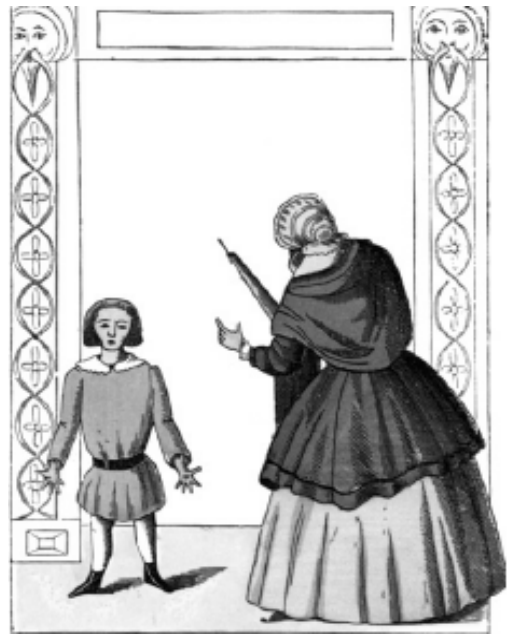

(HOFFMANN 2010:22)

Die Mutter als unübertroffenes Vorbild darzustellen, mag in Złota różḋka Anliegen des Autors sein. Auch in Pawetek Wiercipięta kommt das Wort ,Mutter' in der Koseform ,mamunia‘ vor, was allerdings im krassen Gegensatz zur parallel auftretenden Bezeichnung ,ojciec‘ (und nicht die möglichen Koseformen ,tata“ oder ,tatuś') steht. Die Geschichten Jędruś Gap [Hans Guck-in-die-Luft] und Straszny przypadek z parasolem [Die Geschichte vom fliegenden Robert; wörtl. ,Der schreckliche Fall mit dem Regenschirm‘] können als Belege dafür gelten, wie sehr man sich an der Meinung der Mutter orientiert und auf sie hört. Folgsam sind im Übrigen auch Tiere, wie etwa die 
mit ihren Pfoten drohenden Katzen, die Paulinchen an die Worte der Mutter erinnern: „,Die Mutter hat's verboten! // Miau! Mio! Miau! Mio! // Wirf’s weg! Sonst brennst du lichterloh!““ (HofFMANN 2010:13)

Obwohl das Auftreten der Mutter eine Gelegenheit für den ,großen Erziehungsauftritt‘ bietet, werden sowohl die Ideen als auch die Chancen, sich dem eigenen Kind anzunähern und auf seine Sichtweise einzugehen, verpasst. Sowohl dem Daumen lutschenden Juleczek wie dem die Suppe verweigernden Michaś gelingt es nicht, dem pädagogischen Zeigefinger der Mutter zu entrinnen. In beiden Fällen spricht die Mutter über den Kopf des Kindes hinweg, ohne auf dessen stummen Hilfeschrei, sein Ringen um Anerkennung und Beachtung, aufmerksam zu werden. Nicht einmal in Gefahrensituationen, wie etwa beim am Tisch zappelnden Pawełek Wiercipięta oder dem sich zu Tode hungernden Michaś, springt sie über ihren Schatten. Dass die verweigerte Kommunikation den Protest des Kindes verschärft, ist naheliegend. Die rücksichtslose Sturheit der Mutter mag begründet sein, denn noch immer wird Jungen keine offene Haltung vorgelebt.

Bei genauerem Hinsehen fällt auf, dass beide Fassungen des Struwwelpeters in ähnlicher Weise aufgebaut sind. Die Texte stellen ein Geschichtenkorpus dar, das als Abfolge von Ereignissen betrachtet werden kann, die in eine Kette von Ursache und Wirkung, Mitteln und Zielen eingebettet sind. Im Hinblick auf den Textaufbau stößt man jedoch auf einen latenten Konflikt, der zwischen dem Vorspruch und dem Haupttext besteht. Das Paradoxon liegt darin, dass im Translat in eher lapidarer Form in den Gesamttext eingeführt wird und erst die einzelnen Geschichten für die Textaussage arbeiten. Im Original ist das Gegenteil der Fall. Hier werden dem Leser bereits zu Beginn explizit die guten Seiten gehorsamen und braven Benehmens vor Augen geführt. Es wird sogar dazu aufgefordert, folgsam zu sein, wofür der restliche Text nur Belege liefert. In Konsequenz dessen huldigt der Gesamttext des Originals der im Vorwort ausführlich dargelegten Auffassung musterhaften Verhaltens. Die Diskrepanz, die beide Fassungen diesbezüglich aufweisen, wirkt sich zweifelsohne auf die Textrezeption aus. Während das Vorwort des Originals zu bravem Verhalten auffordert und dessen Vorteile in den Vordergrund rückt, werden in der Einleitung der polnischen Fassung die Konsequenzen tadelnswerten Verhaltens fokussiert und die unglücklichen Geschichten der Kinderfiguren vorweggenommen, was in beiden analysierten Fällen entsprechend einen ermutigenden oder entmutigenden Charakter der Textwiedergabe implizieren kann. Zur Verdeutlichung mögen folgende Stellen aus den Einleitungen dienen: 


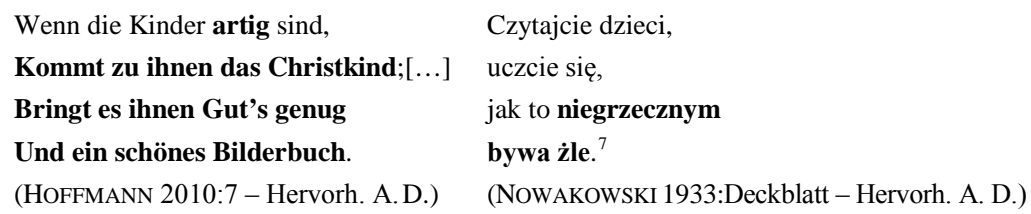

In beiden Texten ist die Verwendung von Komposita auffällig. Diese sind mittlerweile zu festen Wendungen geworden, wie beispielsweise Struwwelpeter, Suppen-Kaspar, Zappel-Philipp, Hans Guck-in-die-Luft für das Deutsche und Staś Straszydto [Der Struwwelpeter] oder Jędruś Gap [Hans Guck-indie-Luft] für das Polnische. Ihr Zweck ist, darauf aufmerksam zu machen, dass die Namensträger von der Norm abweichen. Verachtenswert ist demnach, wer die Haarpflege vernachlässigt oder die Nahrungsaufnahme verweigert, wer bei Tisch hin und wieder mit dem Stuhl wippt oder wer ständig in den Himmel blickt statt auf den Boden und somit den Kontakt zur Wirklichkeit zu verlieren droht. Das heißt, die Abweichung von der Norm ist eine negative Abweichung, daher tragen die Komposita zur Abwertung der betreffenden Kinderfigur bei.

Der polnische Text weicht insofern vom Original ab, als er ausgiebig auf Diminutivformen (vorwiegend bezüglich der Kindernamen) zurückgreift und auf Gott Bezug nimmt. Damit soll zweierlei erreicht werden: Einerseits handelt es sich hier um den Appell, auf tadelnswertes Benehmen zu verzichten, weil es nicht gottgefällig sei, andererseits wird dadurch aber auch Gott als Erziehungsinstanz inszeniert:

Bez przyczyny dręczyć - grzech! ${ }^{8}$

(NowAKOWSKi 1933: Przygoda złego Józia ${ }^{9}$ - Hervorh. A. D.)

Rzuć zapałki, bój się Boga! ${ }^{10}$

(NOWAKOWSKI 1933: Okropna historia z zapatkami ${ }^{11}$ - Hervorh. A. D.)

Bo kara Boża,

Tam, gdzie swawola:

Nie ma Grzegorza,

7 „Lest die Geschichten, Kinder, // lernt recht brav, // wie es Unartigen // manchmal schlecht ergeht." (Hervorh. A. D.)

8 „Grundlos quälen, das ist Sünde!“ (Hervorh. - A.D.)

$9 \quad$ Die Geschichte vom bösen Friederich; wörtl. ,Das Abenteuer des bösen Józios‘.

10 „Wirf, um Gottes willen, die Streichhölzer weg!“ (Hervorh. A.D.)

11 Die gar traurige Geschichte mit dem Feuerzeug; wörtl. ,Die furchtbare Geschichte mit den Streichhölzern`. 
Anna Daszkiewicz

\author{
Ni parasola! ${ }^{12}$ \\ (NOWAKOWSKI 1933: Straszny przypadek z parasolem ${ }^{13}$ - Hervorh. A.D.) \\ I choć krzyczą wniebogłosy \\ Olbrzym na to nie uważa. ${ }^{14}$ \\ (NowAKOWSKI 1933: Czarne dzieci ${ }^{15}$ - Hervorh. A.D.)
}

Ein weiterer Unterschied zwischen dem Original und der Übertragung betrifft die Bilddarstellung: Zwar dient auch in der polnischen Fassung das Bild als Textergänzung und Illustration der Aussage zugleich. Allerdings schlägt die polnische Fassung den Originaltext in Dynamik und Grausamkeit des Bildhaften, was sich beispielsweise in dem folgenden Parallelbild zeigt:
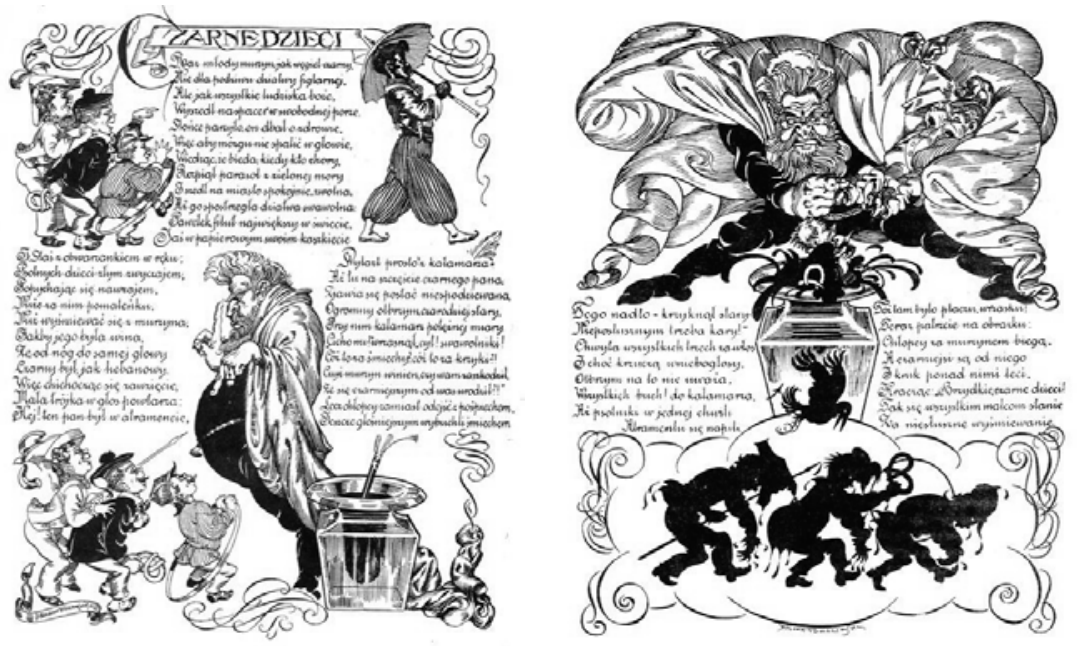

(NOWAKOWSKI 1933: Czarne dzieci)

12 „Denn Gottes Strafe ist dort // wo man ausgelassen ist: // Es gibt weder Grzegorz // Noch seinen Regenschirm!“ (Hervorh. A.D.)

13 Die Geschichte vom fliegenden Robert; wörtl. ,Der furchtbare Fall mit dem Regenschirm‘.

14 „Und auch wenn sie zum Himmel schreien, // lässt es den Riesen kalt. (Hervorh. A.D.)

15 Die Geschichte von den schwarzen Buben; wörtl. ,Schwarze Kinder‘. 


\section{Zur kritischen Auseinandersetzung mit dem Original: FRIEDRICH KARL WAECHTERs Der Anti-Struwwelpeter}

Der Struwwelpeter und sein kritischer Nachfolger weisen sowohl Gemeinsamkeiten als auch Unterschiede auf. Die Autoren beider Werke geben musterhaftem Verhalten eine zentrale Position im Text. In beiden Werken wird zudem der Definition und Betonung vorbildlichen Benehmens ein ausgebautes und in sich stimmiges Argumentationsverfahren untergeordnet. ${ }^{16}$

Dabei wird Hoffmanns Konzept, ,„artig“ mit gehorsam gleichzusetzen ${ }^{17}$, von Waechter als bedenklich wahrgenommen und kritisch hinterfragt. ${ }^{18}$ Waechter verweist auf das Defizit des im Original propagierten Erziehungsideals, demzufolge Kindern weder Autonomie noch Unabhängigkeit zusteht, dafür aber

16 Während im Original den Geschichten eine präzise Erläuterung mustergültigen Verhaltens vorangestellt ist (was die Analyserichtung des Textes vorgibt), wird die Erläuterung im Anti-Struwwelpeter gezielt an das Ende des Textes verschoben. Waechters Vorgehen macht eine vorschnelle Beurteilung von Handlungen der Figuren unmöglich und erlaubt es nicht, die Pointe vorwegzunehmen.

17 Vgl. dazu HeIDENREICH (2009:29): „Artig sein! Das ist das Wichtigste, das man von Kindern verlangt, und artig heißt nichts anderes als: den Großen nicht lästig sein, sich nach ihren Vorstellungen benehmen."; vgl. auch Und die Mutter blicket stumm... 150 Jahre Struwwelpeter (1994:32f.): „Um Verhaltensregulierungen geht es auch im ,Struwwelpeter'. Der garstige Struwwelpeter soll vorführen, daß Kinder verlacht, stigmatisiert und zum Außenseiter gemacht werden, wenn sie sich dem Ideal von Ordnung und Sauberkeit widersetzten. [...] ,Fromm und brav', das war das Kindheitsideal nicht nur des 19. Jahrhunderts. Kinderbücher, in denen Kinder ,froh und frei‘ sein durften, waren die Ausnahme. In der Regel rief ein jeder ,Pfui“, wenn Kinder froh und frei das taten, was ihnen Freude machte.“

18 Mit dem Anti-Struwwelpeter mag Waechter mit der Disziplinierungstradition der Kinderbücher aus dem 19. Jahrhundert (wo unter dem Deckmantel der pädagogischen Notwendigkeit allerlei elterliche Straffantasien ausgetobt wurden) gebrochen haben. Vgl. dazu Gruntz-Stoll (2009:173): „[...] von Strafen oder Katastrophen ist hier nicht mehr die Rede, und wenn jemand an den Pranger gestellt wird, so sind es jene Erwachsenen, die noch nicht gemerkt haben, dass die Zeiten nicht mehr dieselben sind wie vor Jahren und Jahrzehnten. Statt von Kinderfehlern oder Problemverhalten wird - zumindest vorübergehend - von Verhaltensoriginalität gesprochen und jede Form von pädagogischer Repression zumindest offiziell - abgelehnt.“ 
Unterwürfigkeit erwartet wird. ${ }^{19}$ Aus diesem Grund orientiert sich der Autor des Anti-Struwwelpeters bei der Formulierung seines Erziehungskonzeptes am Gegenteil. Dazu greift er allerdings auf den ursprünglichen Stoff zurück, unterzieht ausgewählte Textpassagen einer aufwendigen Analyse und weiß sie in sein Textganzes sachkundig und sachbezogen einzuflechten. Zwar wird dem Bezugstext nicht selten das Grundkonzept entlehnt, dieses wird jedoch ausgebaut und mit doppelbödiger Ironie versehen. Die Erziehungskonzepte Hoffmanns und Waechters sind überwiegend konträr, wobei WAECHTERs Anti-Struwwelpeter die Rolle der kritischen Auseinandersetzung mit dem Originalwerk zukommt. ${ }^{20}$

Im Grunde genommen wird im Anti-Struwwelpeter darauf verwiesen, wie schwach die Position der Erwachsenen sein kann, wenn sie ihre Autorität missbrauchen. Alle erwachsenen Helden versuchen, ihrem etwas nassforschen Nachwuchs ein Unterlegenheitsgefühl einzuflößen, um Gehorsam und Respekt zu erzwingen. Es zeigt sich jedoch, dass es beim Versuch bleibt, denn ihre Kinder lassen sich weder einschüchtern noch provozieren. ${ }^{21}$

Alle Kinderfiguren des Anti-Struwwelpeters sind selbstsicher und durchsetzungsfähig. Im Gegensatz zu den Protagonisten Hoffmanns können sie ihre Wünsche und Bedürfnisse frei artikulieren. Sie scheinen optimistisch eingestellt und im Einklang mit sich selbst zu sein. Denn Klugheit wird im AntiStruwwelpeter in erster Linie mit Fröhlichkeit und Freiheit assoziiert, abge-

19 Vgl. dazu CARCENAC-LECOMTE (2009:136): „Moralisierend stellt Waechter nun nicht mehr wie bei Hoffmann die übertriebenen Folgen des Unartigseins dar, sondern die tatsächlichen psychischen Folgen des Angepaßt- und Artigseins.“

20 Vgl. Von Struwwelhitler bis Punkerpeter. Struwwelpeter-Parodien vom Ersten Weltkrieg bis heute (1998:30): „Die Idee zum Anti-Struwwelpeter entstand in einer Gruppe beim Insel Verlag, die sich vornahm, anti-autoritäre Bücher für Kinder zu machen. Die Struwwelpeter-Bearbeitung war eines der ersten Projekte; nicht weil Struwwelpeter als besonders autoritär und verdammungswürdig erschien, sondern weil es reizte, sich an einem so überaus bekannten Buch zu reiben."

21 Es ist dabei erwähnenswert, dass die kindlichen Helden Waechters eher im Kollektiv auftreten, während sie bei Hoffmann meist vereinzelt auftreten. Vgl. dazu Von Struwwelhitler bis Punkerpeter. Struwwelpeter-Parodien vom Ersten Weltkrieg bis heute (1998:31): „Im Kinderkollektiv, in fantasievoller Wahl ihrer Mittel, sind Daumenlutscher, Friedrich und Pauline stark genug, der Repression der Erwachsenen zu begegnen und die Oberhand zu gewinnen. Gelingt es ihnen, die Erwachsenen auszumanövrieren, sind die Kinder fähig, sich eine eigene Welt zu schaffen, die durch Gleichheit, Solidarität und lustvolles Spiel geprägt ist.“ 
zielt wird auf Autonomie und Selbstzufriedenheit. Im Zusammenhang damit lässt Wächter seine kindlichen Helden eigeninitiativ agieren und den elterlichen Ermahnungen zuwiderhandeln, falls sie die Kinder davon abhalten, nach Persönlichkeitsintegration zu streben. Damit wird jedenfalls die Entfaltung des aufkeimenden Ichs gefördert, die im ursprünglichen Text immer wieder gehemmt wurde. Die Selbstsicherheit der Kinderfiguren im AntiStruwwelpeter zeigt sich auch darin, dass sie weder vernachlässigt ${ }^{22}$ noch betrübt wirken, selbst wenn sie von ihren Eltern unbeaufsichtigt sind. Das betrifft in erster Linie den Anti-Struwwelpeter selbst, dessen Nägel geschnitten sind und dessen Haar stark gelockt ist, was jedes Anzeichen von Ungepflegtsein endgültig aufhebt:

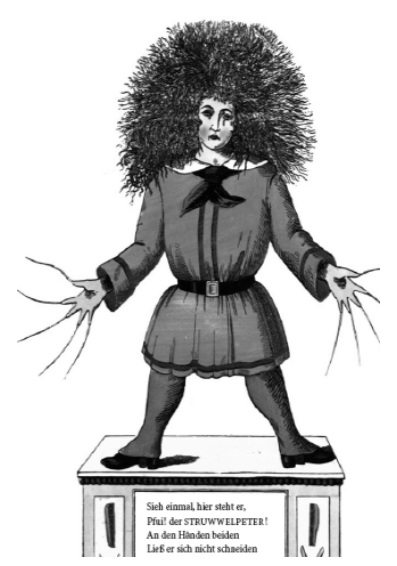

(HofFMAnN 2010:9)

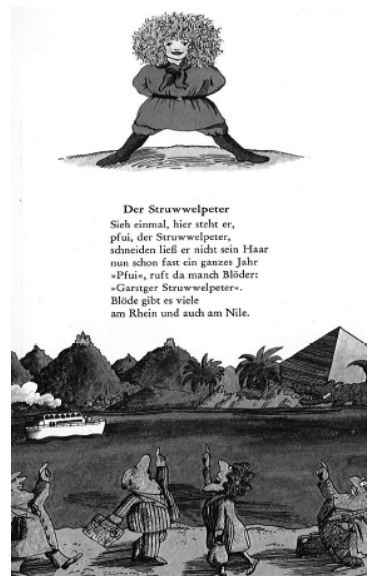

(WAECHTER 1982:9)

22 Darauf macht beispielsweise ECKSTAEDT (1998:39f.) aufmerksam, indem sie ihren Blick auf die Struwwelpeter-Figur Hoffmanns lenkt: „Ein Kind von etwa drei bis vier Jahren, bei dem diese Phase noch nachklingt, so wie man Peter hier sieht, kann sich die Hände und das Gesicht selbst waschen. Aber kein Kind in diesem Alter kann sich selbst die Haare und die Nägel schneiden, und in der Regel werden ihm auch die Haare gekämmt. In diesem Alter braucht das Kind die um sein körperliches Wohl besorgte Person. Vom Haar und den Nägeln abgesehen ist Struwwelpeter sorgfältig und sauber angezogen. Das Anziehen kann ein Kind dieses Alters meist schon allein. So läßt die Diskrepanz zwischen dem, was er selbst nicht kann, und dem, was er kann, auf etwas anderes schließen. [...] Die Nägel sind lange nicht geschnitten. Das heißt, daß dieser traurige Zustand schon längere Zeit besteht.“ 
Kinder und Erwachsene begegnen sich bei Waechter also in einer asymmetrischen Beziehung, wobei die Erwachsenen im Kontrast zu den Kindern dargestellt werden. Die Erwachsenen im Anti-Struwwelpeter lassen sich von Vorurteilen leiten und wittern stets Gefahr (wie im Fall von Paulinchens Eltern, die dem Mädchen das gemeinsame Spiel mit dunkelhäutigen Jungen, den „Mohrenbuben“, untersagen; WAECHTER 1982:13-16). Dazu sind die ElternFiguren bei Waechter selbstbezogen und geldgierig, wie im Fall vom Vater des fliegenden Roberts, der seinen Sohn zum Fliegen gegen Geld auffordert. Sie bleiben ihren verkrusteten Ansichten treu, statt sie zu revidieren. So will etwa der Vater des Suppen-Kaspars dem Sohn seinen Willen aufzwingen, indem er ihm Brot als Nahrungsmittel verweigert und ihm stattdessen immer wieder Suppe geben will.

Im Übrigen wird im Anti-Struwwelpeter jede Art schablonenhaften Verhaltens kritisiert. Die Interjektion „pfui“, die im Original „ein jeder“ (HofFMANN 2010:9) nutzte, um den Titelhelden zu bewerten (und somit seine Position erheblich zu schwächen), und die in Bezug auf die Figur berechtigt schien, wird im Anti-Struwwelpeter lediglich von den „Blöden“ genutzt, die ihren Vorurteilen verhaftet bleiben. Somit wird der bereits angesprochene Ausruf „pfui“ nicht mehr zur Verurteilung des Titelhelden ${ }^{23}$, sondern vielmehr zu seiner Verteidigung verwendet. Auf diese Weise wird eine intolerante und feindselige Verhaltensweise enthüllt, hervorgehoben und angeprangert. Zugleich fordert der Anti-Struwwelpeter zur gegenläufigen Persönlichkeitsentfaltung auf, die auf Selbstsicherheit und der Entwicklung der eigenen Urteilskraft basiert, um zu einer eigenen Weltanschauung und dem damit

23 „Durch den Ausruf ,pfui“ trennt dieser Satz scharf zwischen dem Leser und demjenigen, von dem erzählt wird. Die Interjektion ,pfui` beinhaltet die äußerst affektvolle Zuweisung einer Bedeutung. Nachdrücklich und wie selbstverständlich vollzieht dieser Ausruf die Trennung zwischen ich und du, oder mir und ihm, dem Struwwelpeter. Der Struwwelpeter gerät so in eine Position der Gegenüberstellung. Von Beginn an wird der Leser verführt zu denken: ,Ich doch nicht' oder ,Gott sei Dank, daß ich nicht so bin wie er'. Es erscheint als ein Glück, daß er all das tut, was ich nicht tun soll, daß ihm all dies geschieht, wovon ich wünsche, daß es mir nicht geschehe und zustoße. In Wahrheit ist all das genauso in mir und kann mir genauso passieren wie ihm. Die affektive Steigerung im ,pfui‘ führt fast zu der triumphalen Vergewisserung: ,Nicht ich, sondern du!'. Diese projektive Zuweisung bedeutet eine Konkretisierung alles Bedrohlichen, Schlechten oder Bösen außerhalb meiner selbst im Gegenüber, hier also im Struwwelpeter.“ (WAECHTER 1982:8f.) 
einhergehenden Verhalten zu finden sowie sich nicht dem Diktat der Öffentlichkeit auszusetzen:

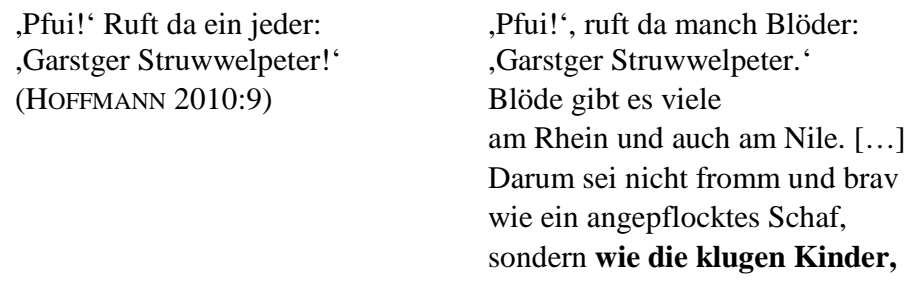

Es sollte allerdings betont werden, dass sowohl im Original als auch in seiner Kritik Argumente für den gewählten Erziehungsstil sowohl im Text selbst als auch in den Bildern zum Ausdruck kommen. Da Waechters Kinderfiguren das Gegenstück zu ihren Eltern sind, werden sie nicht mehr wie im Original als kleine Erwachsene porträtiert ${ }^{24}$ und dürfen auf die „Insignien“ des Kleidungsstils, die zu Hoffmanns Zeit üblich waren (wie beispielsweise Hauben, Blusen mit Jabot aus Spitze oder Gehröcke) verzichten. Stattdessen betont Waechter den zeitgenössischen Kleidungsstil. Dazu schlägt sich Waechters Ironie im Gesichtsausdruck seiner Helden nieder - bei Menschen wie bei Tieren. Alle Figuren des Anti-Struwwelpeter werden demnach grotesk, als eine Karikatur ihrer selbst (meist durch eine etwas zu große Nase) dargestellt. Im Übrigen wirken die männlichen Erwachsenen riesig, sie sind auch durch das groteske Gesicht (durch den etwas zu großen ovalen Kopf und den im Zorn aufgerissenen Mund) gekennzeichnet.

24 Vgl. HerrmanN (1993:32): „Daß man noch bis ins 17. und 18. Jahrhundert Kinder nicht als Kinder, sondern als ,kleine Erwachsene‘ wahrgenommen habe, gehört heute zum festen Bestand historisch-pädagogischer Legendenbildung. Ein Blick auf die niederländische und spanische Genremalerei etwa des 16. Jahrhunderts belehrt sofort eines Besseren. Daß man Kinder und junge Leute ,von Stand` auch noch im 18. Jahrhundert in Kleidung und Habitus so portraitierte, als seien sie ,kleine Erwachsene‘, verstand sich von selbst: als Standesperson nämlich und nicht als Kinder sollten sie gezeigt werden (auch wenn man ihnen häufig die ,Insignien“ ihres Kindseins, wie Spielsachen usw., beigab).“ (Hervorh. im Original - A. D.) 
Anna Daszkiewicz

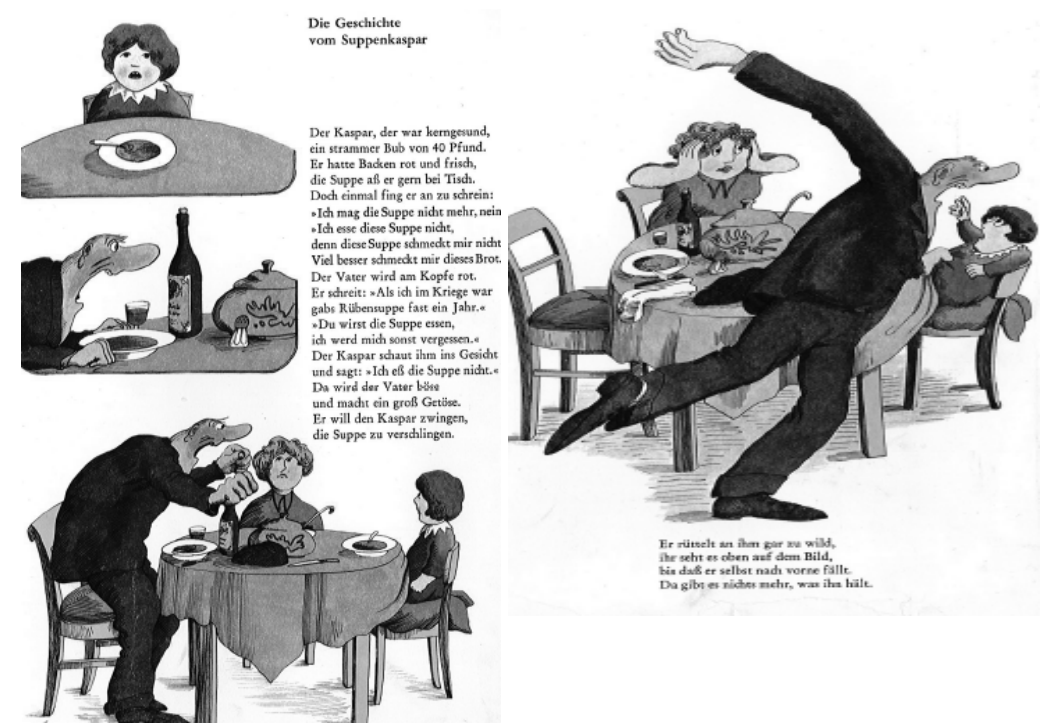

(WAECHTER 1982:18f.)

Im Unterschied zu ihren Eltern werden die kindlichen Helden vorwiegend als fröhliche und freundliche Gestalten inszeniert. Auf ihre Schelmengesichter und die leicht rebellische Natur verweist der diesmal zum Lachen geöffnete Mund:

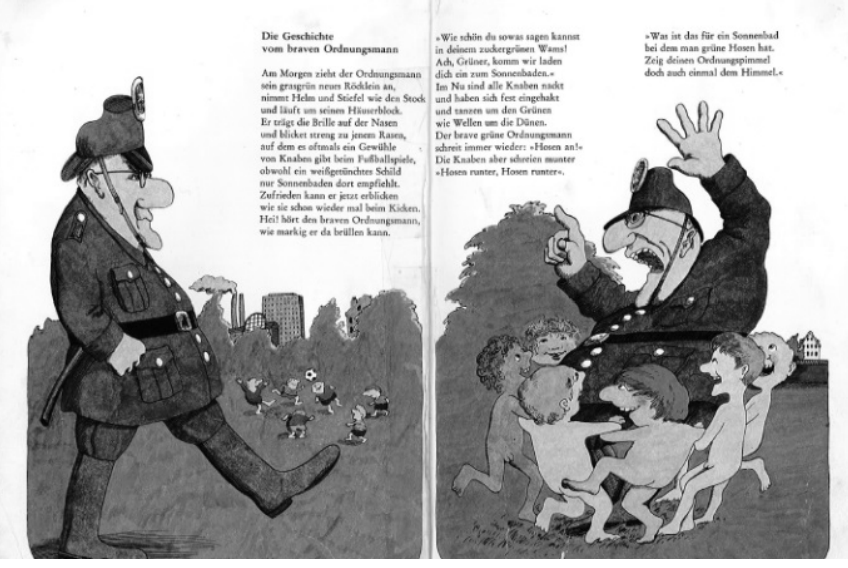

(WAECHTER 1982:10f.) 


\section{Zur Veranschaulichung der Untersuchungsergebnisse}

Gegenstand meiner Untersuchung ist die Rezeption der Text- und Bildfunktion im Struwwelpeter und seinen Nachfolgern von Studierenden des ersten, zweiten und dritten Studienjahrs am Institut für Germanistik der Universität Danzig. An der Untersuchung beteiligten sich ca. 60 Personen. ${ }^{25}$ Sie verfassten Texte, von denen ein Teil in dieser Untersuchung berücksichtigt werden konnte. Die zitierten Texte wurden stilistisch und grammatisch leicht korrigiert, die inhaltliche Aussage blieb unverändert.

Ziel der Untersuchung war, zu prüfen, wie Fremdsprachenlernende ausgewählte Fassungen des Struwwelpeters wahrnehmen, verstehen, interpretieren und was ihnen besonders auffällt. Die Befragten äußerten ihre Meinung frei und ohne vorausgehende Steuerung. Der Vielfalt an Stellungnahmen zum Struwwelpeter hat die Untersuchung ihre konstruktiven, innovativen und nützlichen Ergebnisse zu verdanken.

Die folgende Tabelle veranschaulicht die Untersuchungsergebnisse:

\begin{tabular}{|l|l|}
\hline & Der Struwwelpeter \\
\hline & $\begin{array}{l}\text { Wenn man nun die Bilder betrachtet, die zu jeder Geschichte hinzugefügt } \\
\text { wurden, sieht man, dass sie gemalt wurden, um die Erzählung zu verdeutli- } \\
\text { chen. So sieht man zum Beispiel an der Geschichte vom bösen Friederich (S. } \\
\text { 3-5), welchen Schaden er angerichtet hat und wie er dafür bestraft wird. } \\
\text { Doch das Vereinfachen des Verständnisses ist nicht die einzige Funktion, die } \\
\text { die Bilder haben. Wie man deutlich erkennt, sind manche Bilder recht er- } \\
\text { schreckend (zumindest für Kinder). Nehmen wir als Beispiel Die gar trauri- } \\
\text { ge Geschichte mit dem Feuerzeug. Hier verbrennt das kleine Mädchen, weil } \\
\text { es mit Streichhölzern gespielt hat, und am Ende bleibt von ihm nur noch ein } \\
\text { Häuflein Asche übrig. Ebenso abschreckend sollen die Bilder vom } \\
\text {,Daumenlutscher` sein. Denn welches Kind stellt sich schon ein anderes Kind } \\
\text { mit abgeschnittenen Daumen vor? Alles in allem sollen die Darstellungen } \\
\text { Kinder warnen und sie zum Nachdenken über ihr Benehmen veranlassen. } \\
\text { [...] Vergleicht man nun Text und Bild, sind Ähnlichkeiten und Parallelen zu }\end{array}$ \\
\hline
\end{tabular}

25 Die Befragten bestätigten ihre Einwilligung zur Verwendung der Texte für wissenschaftliche Zwecke schriftlich. Mein Dank gilt besonders den Studierenden für ihre Bereitschaft zur Teilnahme an der Untersuchung und für ihren Arbeitseinsatz. Ohne ihr Engagement wäre diese Untersuchung nicht möglich gewesen. 
erkennen. So verbildlichen die Zeichnungen das, was im Text steht bzw. sind die Kernaussage. Man sieht zum Beispiel in der Geschichte vom bösen Friederich das ganze Ausmaß seiner zerstörerischen Kraft. Die Bilder haben eine Reihenfolge, der man ebenso gut wie der Reihenfolge im Text folgen kann. Dies sieht man vor allem in der Geschichte mit dem Feuerzeug sehr gut, denn auf jedem Bild werden die Katzen gezeigt, wie sie Paulinchen mit den Pfoten drohen. Ich würde nicht sagen, dass die Bilder mehr aussagen als der Text oder dem Text etwas vorwegnehmen. Sie sind lediglich eine Ergänzung der Geschichte und dramatisieren alles noch ein bisschen mehr. (Caroline K., 1. Studienjahr)

Hoffmann hat eine originelle Technik angewandt. Er hat Text und Bild verbunden. Beide sind gleichrangig und spielen eine große Rolle. Die Zeichnungen zeichnen sich durch hohe Qualität und Sorgfalt aus. Sie wurden mit derselben Feder und Tinte wie der Text gezeichnet. Mit absoluter Sicherheit unterstützen sie den Inhalt der Geschichten. Die Bilder stellen das dar, was der Autor geschrieben hat. Man versteht sofort, worum es geht. Das ist sehr wichtig für Kleinkinder, die noch nicht lesen können. (Sara D., 1. Studienon jahr)

Im Struwwelpeter sind Bild- und Textgeschichten ineinander verflochten. (Emilia B., 1. Studienjahr)

Einerseits besteht kein Zweifel daran, dass zwischen Bild und Text eine enge Wechselbeziehung besteht. Man kann hier von einer Bild-Text-Architektur sprechen. Es geht darum, dass Bilder zur Erläuterung oder Kommentierung des Textes dienen. Was man lesen kann, ist auch plastisch dargestellt. Andererseits muss man die Tatsache berücksichtigen, dass die Bilder [im Vergleich mit dem Text] die größere Rolle spielen. (Aleksandra S., 1. Studienjahr)

Die Bilder sind vielsagend, sie allein erzählen eine Geschichte und wirken sich sehr stark auf den Leser aus. Man kann denken, dass die Bilder mehr als der Text sagen und dass sie, und nicht der Text, primär sind. (Aleksander O., 1. Studienjahr)

Dem Text wird jedenfalls die Illustration in Form der detaillierten Bilderfolgen beigefügt. Wären dem Text keine Illustrationen beigefügt, würden sich die Geschehnisse noch schärfer und krasser zugleich auf die Rezipienten auswirken. (Stefanie O., 1. Studienjahr)

Kinder haben zwar viel Fantasie, aber die Bilder prägen sich ihnen ein weiteres Mal ins Gedächtnis ein. Des Weiteren vermitteln die Bilder eine gewisse Atmosphäre der Angst und des Verbotenen. Das Kind fühlt mit den Kindern, die bestraft worden sind, freut sich aber gleichzeitig, nicht an ihrer Stelle zu sein und versteht und lernt somit eigenständig, in Zukunft nicht so zu handeln wie die Protagonisten der Kurzgeschichten Hoffmanns. (Marianne K., 1. Studienjahr) 
Der Anti-Struwwelpeter

Auch beim Anti-Struwwelpeter sind die Bilder eine Widerspiegelung des Textes. Sie unterstützen den Inhalt und bereichern die Geschichten. Allerdings sind die Zeichnungen viel farbiger und nicht mehr so drastisch wie im Struwwelpeter. Man könnte sie lustig nennen. Sie wirken nicht abschreckend. Es gibt hier keine Übertreibung, Aufbauschung. (Sara D., 1. Studienjahr)

Im Anti-Struwwelpeter sind die Bilder nicht so extrem wie im Struwwelpeter, wo dem Daumenlutscher die Daumen abgeschnitten werden und Paulinchen verbrennt. Die Bilder des Anti-Struwwelpeters sind irgendwie fröhlicher und ,runder'. Außerdem moderner (weil das Werk in einer anderen Zeit entstanden ist). Im Anti-Struwwelpeter lachen die Kinder mehr, sind fröhlicher. Die Geschichte vom Ordnungsmann erinnert an FKK (vielleicht weil es die Zeit war, in der alles legalisiert war und alle gleichberechtigt waren). Im Struwwelpeter sind die Bilder ,eckiger‘, trauriger und etwas älter (vielleicht wirken sie deshalb makabrer). (Caroline K., 1. Studienjahr)

Im Anti-Struwwelpeter sind die Zeichnungen nicht so makaber wie im Prototyp, wo abgehackte Finger, das verbrannte Paulinchen, der knochendünne Kaspar zu sehen waren. Im Anti-Struwwelpeter sind die Figuren runder bzw. gerundet. Die Geschichte vom braven Ordnungsmann wirkt ein klein wenig pervers; FKK?

(Marianne K., 1. Studienjahr)

Im Gegensatz zum Struwwelpeter gibt es im Anti-Struwwelpeter eigentlich nur noch kinderfreundliche Bilder. Da ist von den vor Schmerzen dahinsiechenden, verstümmelten Kindern keine Spur. Dagegen wird dargestellt, wie sich die Kleinen in schwierigen Situationen zu benehmen wissen, Ruhe bewahren und allen Widrigkeiten im Leben mutig standhalten. (Stefanie O., 1. Studienjahr)

Während die Bilder im Struwwelpeter authentisch sind und die biedermeierliche Realität widerspiegeln, sind die Illustrationen im Anti-Struwwelpeter verzerrt und knallig. (Aleksandra S., 1. Studienjahr)

Im Anti-Struwwelpeter sind die Illustrationen nicht so detailliert wie im Struwwelpeter. Der Titelheld erweckt kein Mitleid mehr. Man sieht sogar ein Lächeln auf seinem Gesicht. Dies besagt allerdings, dass sich seine Funktion und die der anderen Kinderfiguren völlig verändert hat; sie dienen nämlich nicht mehr der Abschreckung. (Żaneta K., 1. Studienjahr) 
Anna Daszkiewicz

Złota różḋ̇ka

Das im Titel Złota różdżka suggerierte Märchenhafte und Magische schlägt sich vor allem in einer sehr geschmückten Schrift- und Bilddarstellung nieder. (Iwona P., 3. Studienjahr)

In der vorliegenden deutschen Ausgabe sind die Illustrationen meist bunt und vermitteln trotz der Tragik mancher Geschehnisse einen recht fröhlichen Ausdruck. Gemeint ist hier beispielsweise der Schneider, der Konrad beide Daumen abschneidet. In der polnischen Ausgabe sind die Bilder schwarzweiß und wie mit der Feder gezeichnet. Sie können auf Kinder etwas furchteinflößender wirken, weil die Figuren etwas strenger aussehen, doch mir persönlich gefallen sie besser als die bunten Bilder der deutschen Ausgabe.

(Adrian J., 2. Studienjahr)

Im Gegensatz zu den ursprünglichen Illustrationen bringen die Bilder der polnischen Fassung nur noch negative Emotionen zum Ausdruck. (Bożena K., 2. Studienjahr)

In der polnischen Struwwelpeter-Bearbeitung sind die Bilder viel grausamer und unmenschlicher als in der Vorlage. (Weronika K., 3. Studienjahr)

In Zlota różdżka sind die Bilder sehr drastisch, noch drastischer und grässlicher als im Struwwelpeter. Dazu sind sie einfarbig, die Gesichtsausdrücke der handelnden Personen verraten viel mehr Schmerz und Ungerechtigkeit als die der Vorlage. Darüber hinaus schlägt sich das Tragische in noch höherem Maße als im Original in den Titeln der Geschichten nieder: Okropna historia z zapalkami ${ }^{26}$; Straszny przypadek z parasolem ${ }^{27}$; Historia bardzo smutna o chłopcu, który nie chciat jeść zupy ${ }^{28}$. (Monika G., 3. Studienjahr)

In der polnischen Version sind die Bilder drastischer als im Original. (Maja Z., 2. Studienjahr)

Die Bilder in der polnischen Version unterscheiden sich von denen des Originals. Zwar sind sie in Bezug auf die Bildsituation sehr ähnlich, aber besonders die Gesichter der Figuren drücken die Emotionen intensiver und schriller aus. Die Bilder sind eindeutig aggressiver, blutiger. Die Position der Helden ist dynamischer. Ich vermute, dass das daran liegt, dass der Zeichner die gefährlichen Situationen und ihre Konsequenzen noch deutlicher zeigen will. (Agnieszka L., 3. Studienjahr)

26 ,Die furchtbare Geschichte mit den Streichhölzern` (Hervorh. A.D.).

27 ,Der schreckliche Fall mit dem Regenschirm‘ (Hervorh. A.D.).

28 ,Die sehr traurige Geschichte vom Jungen, der seine Suppe nicht essen wollte‘ (Hervorh. A.D.). 
In der polnischen Version liegt eine andere Art Bild vor. Im Gegensatz zum Original sind sie dynamischer, außerdem blutig und hart. (Dawid K., 2. Studienjahr)

Für die polnische Version ist gerade eine gewisse Dynamik charakteristisch. Die Bilder sind gefühlvoll. Die Kindergesichter können die Rezipienten entsetzen oder sogar erschrecken. Dazu haben die Bilder in der polnischen Version keine rechten Winkel, die Linien sind knotig, schief. (Alicja O., 2. Studienjahr)

Da der Inhalt des Struwwelpeters in jeder der drei besprochenen Ausgaben in einer bildlichen Realität verortet ist, verwundert die Befragten die Aufgabe, sich zu den Wort-Bild-Bezügen zu äußern, nicht. Die Teilnehmenden der Untersuchung kommen zu dem Schluss, dass Sprache und Bild untrennbar miteinander verknüpft sind, ja sogar verschmelzen (es wird sogar von „TextBild-Architektur“ gesprochen). Bemerkenswert dabei ist, dass Sprache und Bild von den Befragten nicht als sich ergänzende Teiltexte, sondern als separate Gesamttexte wahrgenommen werden, die sich zwar überlappen, aber auch getrennt Kontextualität und Funktionalität entwickeln können. Daher partizipieren beide Zeichensysteme gleichermaßen am Dekodierungsprozess der Textaussage und werden zu integrativen Teilen des Gesamttextes.

Obwohl die Grenzen zwischen der schriftlichen und der bildlichen Textwelt fließend sind, tendieren die Rezipierenden beim Struwwelpeter dazu, dem Bild, das ursprünglich als Alternativangebot für die Leser gedacht war, potenzielle Mehrdeutigkeit zuzuschreiben. Den Aussagen der Befragten ist direkt zu entnehmen, dass die dem Text beigefügten Illustrationen Mehrdimensionalität aufweisen. Dementsprechend wirken sie kommunikationsfähig, emotionsgeladen (sie sind Fokusmarker und Emotionsträger, indem sie dem Gesamttext, abhängig von der zu vermittelnden Botschaft, einerseits einen dramatischen und makabren, andererseits aber stellenweise auch einen grotesken und fröhlichen Ausdruck verleihen, was entsprechend durch die „runden“ bzw. „gerundeten“ oder „eckigen“ und „knotigen“ Konturen der Hauptfiguren hervorgehoben wird), dynamisch, verständniserleichternd und einprägsam. Der entscheidende Vorteil der Illustrationen liegt zudem darin begründet, dass sie das Anliegen des Autors veranschaulichen - sie eignen sich nämlich perfekt zur Warnung. Das hat eine leichtere Generierung des Texttragenden und der Entschlüsselung der Gesamtbotschaft zur Folge.

Die Neigung mancher Befragten, die Verbildlichung und nicht die Versprachlichung der Sachverhalte bei der Wahrnehmung des Textganzen als 
ausschlaggebend zu betrachten, scheint allerdings kaum gerechtfertigt zu sein. Vgl. dazu die folgenden Beispiele:

Man könnte denken, dass die Bilder mehr als der Text sagen und dass sie und nicht der Text primär sind. (Aleksander O., 1. Studienjahr)

Einerseits besteht kein Zweifel, dass zwischen Bildern und Text eine enge Wechselbeziehung besteht. [...] Andererseits muss man die Tatsache berücksichtigen, dass die Bilder die größere Rolle spielen. (Aleksandra S., 1. Studienjahr)

Zwar lässt sich der kodierte Charakter der Illustrationen nicht bestreiten, es darf jedoch nicht vergessen werden, dass sie lediglich einen Ausschnitt der Textrealität darstellen. Die Illustrationen des Struwwelpeters können nur eingeschränkte Perspektiven zeigen, indem sie den Blick innerhalb eines Bildes bündeln. Das gilt auch dann, wenn sie eine Abfolge bilden. Das intuitive Bildverständnis reicht zur Dekodierung der Textbotschaft sicherlich nicht aus. Jedes Bild erfordert eine Präzisierung durch den Text, da die Interpretation sonst nicht gelingen kann: Die Textaussage lässt sich aus der Wahrnehmung einer einzelnen Moment- und Handlungssequenz nicht ablesen, sie geht weit über das Sichtbare hinaus. Zweifellos wird das Gesamtgeschehen konsequent durch immer neue Details präzisiert. Sie liefern den Rezipienten wichtige Antworten und Hintergrundinformationen, auf deren Grundlage sie die Abbildungen weiterdenken können. Reduziert man die Textaussage auf die Bildaussage, so beeinträchtigt dies die Interpretation des Textes oder führt sogar zu einer Fehlinterpretation. Das mag den Befragten allerdings entgangen sein.

Allgemein nehmen die Rezipienten häufiger Stellung zur Bild- als zur Textwahrnehmung. Die Schwerfälligkeit, mit der sie den Text analysieren, rührt in erster Linie daher, dass das Deutsche eine Fremdsprache für sie ist, die sie noch erlernen. Zwar ist ihr Deutsch ausreichend, um komplexere Sachverhalte ausdrücken zu können. Es fehlt ihnen jedoch grundsätzlich das Wissen über die in der Kultur jeder Nation verankerten, präferierten Erziehungsmodelle. Kenntnis über sie erwerben Muttersprachler mit dem Erstspracherwerb. Eine entsprechende Prägung kann die Vorstellungskraft sowie die Orientierung von Fremdsprachenlernenden steuern. Kurz, die Muttersprache hat großen Einfluss auf die Wahrnehmung der in der Zielsprache enkodierten Inhalte. Dass die eventuell nicht verstandenen kulturellen Konventionen der Zielsprache und nicht nur die Fremdsprache das Textverständnis beeinflussen bzw. steuern, ist schwer zu akzeptieren, obwohl im Wesentlichen sie die Ursache für Unterschiede im Weltbild und den Denkmustern (für die Andersartigkeit der Sprachen überhaupt) sind. Dies könnte auch für die drei hier 
analysierten Fassungen des Struwwelpeters gelten. Der auffälligste Unterschied zwischen den drei Versionen besteht darin, dass nur die deutschen Struwwelpeter-Texte ein gewisses Maß an (schwarzem) Humor bzw. Ironie kennzeichnet. Beide deutschsprachigen Versionen scheinen nicht völlig ernst gemeint zu sein, denn Grausamkeit wird konsequent durch humoristische Übertreibung ausgeglichen. Als Belege dafür können die übergroße Frisur des Struwwelpeters, der immer weiter abmagernde Suppen-Kaspar oder der sich dem Daumenlutscher tänzelnd nähernde Schneider mit seiner überdimensionalen Schere gelten. Der Humor wird im deutschen StruwwelpeterStoff darüber hinaus auch durch den zum Händeklatschen, Mitsprechen sowie Weiterreimen animierenden Rhythmus bewirkt, was jedoch beim Transfer ins Polnische nicht berücksichtigt wurde und sich damit als Übersetzungsfalle für die gesamte polnische Struwwelpeter-Bearbeitung erweist. Der Schwerpunkt der polnischen Struwwelpeter-Version liegt grundsätzlich darauf, die jede einzelne Geschichte begleitende Dramaturgie, die in der polnischen Fassung noch vertieft wurde, zu übertragen. Die Schwierigkeit, die Zweideutigkeit der deutschen Gesten mit entsprechenden polnischen Gesten wiederzugeben, führt dazu, dass der Wort-Bild-Charakter in Złota różdżka makaber und abstoßend wirkt. Die Abweichung fällt auch den Studierenden auf, wenn sie auch den Grund nicht benennen können:

Im Gegensatz zu den ursprünglichen Illustrationen bringen die Bilder der polnischen Fassung nur noch negative Emotionen zum Ausdruck. (Bożena K., 2. Studienjahr)

In der polnischen Struwwelpeter-Bearbeitung sind die Bilder viel grausamer und unmenschlicher als in der Vorlage. (Weronika K., 3. Studienjahr)

In Zlota różdżka sind die Bilder sehr drastisch, noch drastischer und grässlicher als im Struwwelpeter-Original. Dazu sind sie einfarbig, die Gesichtsausdrücke der handelnden Personen verraten viel mehr Schmerz und Ungerechtigkeit als die der Vorlage. Darüber hinaus schlägt sich das Tragische in noch höherem Maße als im Original in den Titeln der Geschichten nieder: Okropna historia z zapatkami; Straszny przypadek z parasolem; Historia bardzo smutna o chłopcu, który nie chciat jeść zupy. (Monika G., 3. Studienjahr)

Aus den Ausführungen geht hervor, dass die Arbeit an den Text-BildBezügen in den Struwwelpeter-Versionen nicht nur aufgrund ihres informativen, sondern auch wegen des interkulturellen Charakters von großem Nutzen ist. Durch diese Textauslegung konnte das Wissen über den Text erheblich erweitert werden. Die Analyse der verbalen und nonverbalen Kodes hat wichtige Belege für die wechselseitige Integration von Text und Bild geliefert. Alles in allem wird der Rezipient dafür sensibilisiert, dass die Wahrnehmung des Beobachters nicht nur von seiner aktuellen Verfassung, sondern 
auch von seiner Muttersprache abhängig ist. Der Artikel verweist zudem auf die Rolle des bebilderten Kinderbuches in der Erziehung. Dazu leisten Wort und Bild gleichermaßen einen Beitrag. Beide werden benötigt, um Heranwachsenden einerseits Anschauungsunterricht zu geben und es den Verfassern andererseits zu ermöglichen, ihre eigene Einstellung zur Verhaltensregulierung darzustellen. Dass sich die jeweiligen Verfasser der StruwwelpeterFassungen hinsichtlich ihrer Auffassung von Erziehung unterscheiden, drückt sich in ihrer Wort- und Bildgestaltung aus. Wort und Bild unterliegen permanenten Veränderungen, die wiederum vom Zeitgeist und den damit einhergehenden Erwartungen der Gesellschaft beeinflusst werden. Schließlich liefert der vorliegende Artikel Belege dafür, dass durch diese Phänomene eine nachhaltige Prägung erfolgen kann. Dies verdeutlichen die Untersuchungsergebnisse bzw. die Aussagen der befragten Rezipienten, die die bis heute anhaltende Nachwirkung des ursprünglichen Bilderbuches von Heinrich Hoffmann (1845) bestätigen.

\section{Literatur}

BuRwick, Roswitha (2010): Heinrich Hoffmanns Struwwelpeter. Lustige Geschichten oder schwarze Pädagogik? In: FULDA, DANIEL / ROEBEN, ANTJE / WichaRD, NORBERT (eds.): Kann man denn auch nicht lachend sehr ernsthaft sein? Sprachen und Spiele des Lachens in der Literatur. Berlin, 135-148.

Carcenac-Lecomte, Constanze (2009): Der Struwwelpeter. In: François, Etienne / Schulze, Hagen (eds.): Deutsche Erinnerungsorte. Bd. 3. München, 122, 137.

EcKstaedt, Anita (1988): „Der Struwwelpeter“. Dichtung und Deutung. Eine psychoanalytische Studie. Frankfurt (M.).

FLITNER, ANDREAs (2009): Konrad, sprach die Frau Mama. Weinheim/Basel.

GrosChe, ERWIN (2007): Der tierische Struwwelpeter. München.

GruntZ-Stoll, Johannes (2009): Schreiliesel, Struwwelpeter \& Co. Schwierige Kinder in schwierigen Zeiten? In: MeIER Rey, Christine (ed.): Schwierige Zeiten schwierige Kinder. Von Herausforderungen für Kinder und Jugendliche durch Globalisierung und Neoliberalismus. Basel, 163-176.

HACKs, Peter (2010): Ein Kommentar zu Heinrich Hoffmanns „Der Struwwelpeter“. In: HOFFMANN, 34.

HeIDENREICH, ElKe (2009): Mein Struwwelpeter. Berlin.

Herrmann, Ulrich (1993): Aufklärung und Erziehung. Studien zur Funktion der Erziehung im Konstitutionsprozeß der bürgerlichen Gesellschaft im 18. und frühen 19. Jahrhundert in Deutschland. Weinheim. 
Herzog, Gerhard H. / Herzog-Hoinkis, Marion / Siefert, Helmut (eds.) (1995): Heinrich Hoffmann. Leben und Werk in Texten und Bildern. Frankfurt (M.)/Leipzig.

HeinRich-HoffMANN-MuSEum DER Frankfurter Werkgemeinschaft E.V. (ed.) (1998): Von Struwwelhitler bis Punkerpeter. Struwwelpeter-Parodien vom Ersten Weltkrieg bis heute. Eine Ausstellung des Arbeitskreises „Bürger gestalten ihr Museum “. Frankfurt (M.).

Hoffmann, HeInRICH (2010): Der Struwwelpeter. Lustige Geschichten und drollige Bilder. Originalausgabe für die ganze Familie mit einem Vorwort für Kinder von Walter Sauer und einem Nachwort für Eltern von Ulrich Wiedmann. Neckarsteinach.

KLEIN, REIMAR (2005): Sieh einmal, hier steht er! Struwwelpeters beschädigte Kinderwelt. Frankfurt (M.).

LÜTJE, Julius / Maddalena, Franz (1890): Die Struwwelliese oder Lustige Geschichten und drollige Bilder. Esslingen.

MuSEUMSVERBund SÜDNIEDERSACHSEN (ed.) (1994): Und die Mutter blicket stumm... 150 Jahre Struwwelpeter. Göttingen.

NOWAKOWSKI, WACŁAW (1933): Zlota różdżka. Illustriert von Bohdan Nowakowski. Warszawa.

SCHWEIKLE, GÜNTHER / SchweIKLe, IRMgARD (eds.) (1990): Metzler Literatur Lexikon. Begriffe und Definitionen. Stuttgart.

SPence, Robert / Spence, Philip (1941): Struwwelhitler. A Nazi Story Book by Dr. Schrecklichkeit. Eine Parodie des Original-Struwwelpeter. London.

StOlLe, FerdinAnd (1871): Wie der Struwwelpeter entstand. In: Die Gartenlaube 46:768-770.

WAECHTER, FRIEDRICH KARL (1970): Der Anti-Struwwelpeter. Zürich.

- (1982): Der Anti-Struwwelpeter. Darmstadt. 